PROCEEDINGS OF THE

AMERICAN MATHEMATICAL SOCIETY

Volume 132, Number 5, Pages 1305-1309

S 0002-9939(03)07326-X

Article electronically published on December 18, 2003

\title{
VISCOSITY AND RELAXATION APPROXIMATIONS FOR A HYPERBOLIC-ELLIPTIC MIXED TYPE SYSTEM
}

\author{
YUN-GUANG LU AND CHRISTIAN KLINGENBERG
}

Abstract. To a given system of conservation laws

$$
\left\{\begin{array}{c}
u_{t}+f(u, v, h(u, v))_{x}=0 \\
v_{t}+g(u, v, h(u, v))_{x}=0
\end{array}\right.
$$

we associate the system

$$
\left\{\begin{array}{l}
u_{t}+f(u, v, s)_{x}=\epsilon u_{x x} \\
v_{t}+g(u, v, s)_{x}=\epsilon v_{x x} \\
s_{t}+\frac{s-h(u, v)}{\tau}=\epsilon s_{x x},
\end{array}\right.
$$

which is of mixed type. Under certain conditions, convergence of this latter system for $\epsilon \rightarrow 0$ with $\tau=o(\epsilon)$ is established without the need of stability criteria or hyperbolicity of the left-hand sides of the equations.

\section{INTRODUCTION}

In this paper we study singular limits of stiff relaxation and dominant diffusion for a general hyperbolic-elliptic mixed type system of three equations:

$$
\left\{\begin{array}{l}
u_{t}+f(u, v, s)_{x}=\epsilon u_{x x} \\
v_{t}+g(u, v, s)_{x}=\epsilon v_{x x} \\
s_{t}+\frac{s-h(u, v)}{\tau}=\epsilon s_{x x}
\end{array}\right.
$$

with initial data

$$
(u, v, s)_{\mid t=0}=\left(u_{0}, v_{0}, s_{0}\right) .
$$

The third equation in (1.1) contains a relaxation mechanism with $h(u, v)$ as the equilibrium value for $s, \tau$ the relaxation time, and $\epsilon$ the viscous parameter. That is, the relaxation time $\tau$ tends to zero faster than the diffusion parameter $\epsilon, \tau=$ $o(\epsilon), \epsilon \rightarrow 0$. We establish the following general framework: if there exists an a priori $L^{\infty}$ bound uniform with respect to $\epsilon$ and $\tau$ for the solutions of the Cauchy problem (1.1) and (1.2), then the solution sequence converges to the corresponding equilibrium solution of this system:

$$
\left\{\begin{array}{l}
u_{t}+f(u, v, h(u, v))_{x}=0 \\
v_{t}+g(u, v, h(u, v))_{x}=0
\end{array}\right.
$$

where system (1.3) is strictly hyperbolic and genuinely nonlinear in the sense of Lax. Our results indicate that the convergent behavior of such a limit is independent

Received by the editors February 10, 2002.

2000 Mathematics Subject Classification. Primary 35L65.

(C)2003 American Mathematical Society 
of either the stability criterion or the hyperbolicity of the corresponding inviscid quasilinear system

$$
\left\{\begin{array}{l}
u_{t}+f(u, v, s)_{x}=0 \\
v_{t}+g(u, v, s)_{x}=0 \\
s_{t}+\frac{s-h(u, v)}{\tau}=0
\end{array}\right.
$$

which is not the case for other types of limits considered in [1] and [3]. In other words, suppose system (1.4) is of mixed type and does not allow for a stability criterion (which would be available if (1.4) were hyperbolic). Then our method would still allow for convergence to the equilibrium system (1.3).

More precisely, we have the following.

Theorem 1.1. Suppose that the solution sequence $\left(u^{\epsilon, \tau}, v^{\epsilon, \tau}, s^{\epsilon, \tau}\right)$ of the Cauchy problem (1.1), (1.2) is uniformly bounded in $L^{\infty}$ with respect to the parameters $\epsilon, \tau$. If system (1.3) is strictly hyperbolic, genuinely nonlinear and the function $h(u, v)^{2}$ is an entropy of system (1.3), then there exists a subsequence (still denoted by $\left.\left(u^{\epsilon, \tau}, v^{\epsilon, \tau}, s^{\epsilon, \tau}\right)\right)$ such that

$$
\left(u^{\epsilon, \tau}, v^{\epsilon, \tau}, s^{\epsilon, \tau}\right) \rightarrow(u, v, s), \text { a.e. }
$$

as $\epsilon$ and $\tau$ go to zero, where

$$
s=h(u, v), \text { a.e. }
$$

and $(u, v)$ satisfies system (1.3) in the sense of distributions.

\section{Proof of Theorem 1.1}

Since system (1.3) is strictly hyperbolic and genuinely nonlinear in the sense of Lax, by using DiPerna's framework [2] and the theory of compensated compactness, we could prove that $\left(u^{\epsilon, \tau}, v^{\epsilon, \tau}\right) \rightarrow(u, v)$, a.e. as $\epsilon$ and $\tau$ go to zero if we could prove that

$$
\eta\left(u^{\epsilon, \tau}, v^{\epsilon, \tau}\right)_{t}+q\left(u^{\epsilon, \tau}, v^{\epsilon, \tau}\right)_{x} \text { are compact in } H_{l o c}^{-1}\left(R \times R^{+}\right),
$$

where $(\eta(u, v), q(u, v))$ is any pair of entropy-entropy flux of system (1.3).

We can use the estimates in Lemma 2.1 to get the proof of (2.1).

Lemma 2.1. If the conditions in Theorem 1.1 are satisfied, then $\epsilon\left(u^{\epsilon, \tau}\right)^{2}, \epsilon\left(v^{\epsilon, \tau}\right)^{2}$, $\epsilon\left(s^{\epsilon, \tau}\right)^{2}$ and $\left(s^{\epsilon, \tau}-h\left(u^{\epsilon, \tau}, v^{\epsilon, \tau}\right)\right)^{2} / \tau$ are uniformly bounded in $L_{l o c}^{1}\left(R \times R^{+}\right)$with respect to $\epsilon, \tau$.

In fact, if the conclusions in Lemma 2.1 are true, we can rewrite the first two equations in (1.1) by

$$
\left\{\begin{array}{l}
u_{t}+f(u, v, h(u, v))_{x}=(f(u, v, h(u, v))-f(u, v, s))_{x}+\epsilon u_{x x} \\
v_{t}+g(u, v, h(u, v))_{x}=(g(u, v, h(u, v))-g(u, v, s))_{x}+\epsilon v_{x x}
\end{array}\right.
$$


Then for any pair of entropy-entropy flux $(\eta(u, v), q(u, v))$ of system (1.3), we have

$$
\begin{aligned}
\eta(u, v)_{t}+ & q(u, v)_{x} \\
= & \eta_{u}(u, v)(f(u, v, h(u, v))-f(u, v, s))_{x} \\
& +\eta_{v}(u, v)(g(u, v, h(u, v))-g(u, v, s))_{x} \\
& +\epsilon \eta_{u}(u, v) u_{x x}+\epsilon \eta_{v}(u, v) v_{x x} \\
= & \epsilon \eta(u, v)_{x x}-\epsilon\left(\eta_{u u} u_{x}^{2}+2 \eta_{u v} u_{x} v_{x}+\eta_{v v} v_{x}^{2}\right) \\
& +\left[\eta_{u}(u, v)(f(u, v, h(u, v))-f(u, v, s))\right]_{x} \\
& +\left[\eta_{v}(u, v)(g(u, v, h(u, v))-g(u, v, s))\right]_{x} \\
& +\eta_{u x}(u, v) f_{s}\left(u, v, \beta_{1}\right)(s-h(u, v)) \\
& +\eta_{v x} g_{s}\left(u, v, \beta_{2}\right)(s-h(u, v)) \\
= & I_{1}+I_{2}+I_{3}+I_{4}+I_{5},
\end{aligned}
$$

where $\beta_{i}, i=1,2$, are some values between $s$ and $h(u, v)$. From the estimates in Lemma 2 , we can easily prove that $I_{1}, I_{3}$ are compact in $H_{l o c}^{-1}\left(R \times R^{+}\right)$, and $I_{2}, I_{4}$ and $I_{5}$ are bounded in $L_{l o c}^{1}\left(R \times R^{+}\right)$. Thus the right-hand side of (2.3) is compact in $W_{l o c}^{-1, q}\left(R \times R^{+}\right)$for some exponent $q$, where $1<q<2$. But the left-hand side of (2.3) is bounded in $W^{-1, \infty}$. Therefore, by using Murat's Lemma, we get the proof of (2.1). Since $\left(u^{\epsilon, \tau}, v^{\epsilon, \tau}\right) \rightarrow(u, v)$, a.e., using the last estimate in Lemma 2.1, we have the convergence $s^{\epsilon, \tau} \rightarrow s$, a.e. as $\epsilon$ goes to zero. So the proof of Theorem 1.1 is ended.

Proof of Lemma 2.1. Since system (1.3) is strictly hyperbolic and genuinely nonlinear, then there exists a strictly convex entropy $\eta_{1}(u, v)$. Since $(u, v, s)$ is bounded, we can choose a large constant $C_{1}$ such that the function

$$
p(u, v, s)=\frac{s^{2}}{2}-h(u, v) s+C_{1} \eta_{1}(u, v)
$$

satisfies

$$
\begin{aligned}
p_{u u}(u, v, s) u_{x}^{2}+ & p_{v v}(u, v, s) v_{x}^{2}+p_{s s}(u, v, s) s_{x}^{2} \\
& +2 p_{u v}(u, v, s) u_{x} v_{x}+2 p_{u s}(u, v, s) u_{x} s_{x}+2 p_{v s}(u, v, s) v_{x} s_{x} \\
\geq & C_{2}\left(u_{x}^{2}+v_{x}^{2}+s_{x}^{2}\right)
\end{aligned}
$$

for some constant $C_{2}>0$.

Multiplying system (1.1) by $\left(p_{u}, p_{v}, p_{s}\right)$, we have from (2.4) that

$$
\begin{aligned}
& p(u, v, s)_{t}+p_{u}(u, v, s) f(u, v, s)_{x}+p_{v}(u, v, s) g(u, v, s)_{x} \\
& \quad+\frac{(s-h(u, v))^{2}}{\tau}+\epsilon C_{2}\left(u_{x}^{2}+v_{x}^{2}+s_{x}^{2}\right) \\
& \leq \epsilon p_{x x}(u, v, s) .
\end{aligned}
$$


Since

$$
\begin{aligned}
& p_{u}(u, v, s) f(u, v, s)_{x}+p_{v}(u, v, s) g(u, v, s)_{x} \\
= & \left(C_{1} \eta_{1 u}-h_{u}(u, v) s\right) f(u, v, s)_{x}+\left(C_{1} \eta_{1 v}-h_{u}(u, v) s\right) g(u, v, s)_{x} \\
= & \left(C_{1} \eta_{1 u}-h_{u}(u, v) h(u, v)\right)(f(u, v, s)-f(u, v, h(u, v)))_{x} \\
+ & \left(C_{1} \eta_{1 u}-h_{u}(u, v) h(u, v)\right) f(u, v, h(u, v))_{x} \\
+ & \left(C_{1} \eta_{1 v}-h_{v}(u, v) h(u, v)\right)(g(u, v, s)-g(u, v, h(u, v)))_{x} \\
+ & \left(C_{1} \eta_{1 v}-h_{v}(u, v) h(u, v)\right) g(u, v, h(u, v))_{x} \\
- & h_{u}(u, v)(s-h(u, v)) f(u, v, s)_{x}-h_{v}(u, v)(s-h(u, v)) g(u, v, s)_{x} \\
= & {\left[\left(C_{1} \eta_{1 u}-h_{u}(u, v) h(u, v)\right)(f(u, v, s)-f(u, v, h(u, v)))\right]_{x} } \\
+ & {\left[\left(C_{1} \eta_{1 v}-h_{v}(u, v) h(u, v)\right)(g(u, v, s)-g(u, v, h(u, v)))\right]_{x} } \\
- & \left(C_{1} \eta_{1 u}-h_{u}(u, v) h(u, v)\right)_{x} f_{s}\left(u, v, \beta_{3}\right)(s-h(u, v)) \\
- & \left(C_{1} \eta_{1 v}-h_{v}(u, v) h(u, v)\right)_{x} g_{s}\left(u, v, \beta_{4}\right)(s-h(u, v)) \\
+ & \left(C_{1} q_{1}(u, v)-q_{2}(u, v)\right)_{x},
\end{aligned}
$$

where $q_{1}, q_{2}$ are two entropy fluxes of system (1.3) corresponding to the entropies $\eta_{1}$ and $h^{2}(u, v) / 2$, and $\beta_{i}, i=3,4$ are some values between $s$ and $h(u, v)$. If $\tau$ is much smaller than $\epsilon$, then we have from (2.5) and (2.6) that

$$
\begin{aligned}
& p(u, v, s)_{t}+F(u, v, s)_{x}+C_{3} \frac{(s-h(u, v))^{2}}{\tau} \\
+ & \epsilon C_{4}\left(u_{x}^{2}+v_{x}^{2}+s_{x}^{2}\right) \leq \epsilon p_{x x}(u, v, s),
\end{aligned}
$$

for a function $F(u, v, s)$ and positive constants $C_{3}$ and $C_{4}$ depending on the bounds of the second derivatives of $p$ and the first derivatives of $f$ and $g$.

Multiplying (2.7) by a suitable nonnegative test function and then integrating by parts on $R \times R^{+}$, we get the estimates in Lemma 2.1 .

Acknowledgments. Yun-guang Lu's research is partially supported by a Humboldt fellowship, a grant from the National University of Colombia, Bogota and by the Chinese NNSF 10071080.

\section{REFERENCES}

[1] G. Q. Chen, C. D. Levermore, and T. P. Liu, Hyperbolic conservation laws with stiff relaxation terms and entropy, Comm. Pure Appl. Math. 47 (1994), 787-830. MR 95h:35133

[2] R. J. DiPerna, Convergence of approximate solutions to conservation laws, Arch. Rational Mech. Anal. 82 (1983), 27-70. MR 84k:35091 
[3] A. E. Tzavaras, Materials with internal variables and relaxation to conservation laws, Arch. Rational Mech. Anal. 146 (1999), 129-155. MR 2000i:74004

Department of Mathematics, University of Science and Technology of China, Hefei and Departamento de Matemáticas Universidad Nacional de Colombia, Bogota

E-mail address: yglu@matematicas.unal.edu.co

Department of Mathematicas, Würzburg University, Würzburg, 97074, Germany

E-mail address: klingen@mathematik.uni-wuerzburg.de 